\title{
Aceitação de cultivares de bananas resistentes à Sigatoka Negra junto ao consumidor da região Nordeste do Brasil
}

\author{
Acceptance of banana cultivars resistant to Black Sigatoka by the consumer market of Northeast \\ Brazilian region
}

\begin{abstract}
Deborah dos Santos Garruti' ${ }^{\text {** }}$ Melissa de Lima Matias ${ }^{\text {II }}$ Heliofábia Virgínia de Vasconcelos Facundo ${ }^{\text {II }}$

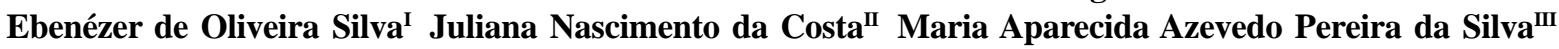

\section{RESUMO}

O Mal da Sigatoka Negra é devastador para as cultivares tradicionais de banana. No Brasil, cultivares resistentes estão sendo desenvolvidas, mas se a preferência do consumidor não for considerada, o programa de melhoramento pode falhar. Neste trabalho, a aceitabilidade de quatro cultivares resistentes foi acessada no Nordeste brasileiro e comparada a quatro variedades comerciais. Dois grupos de consumidores participaram: adultos jovens e donas de casa. Escala hedônica foi aplicada para aparência externa dos cachos, aceitação global e aceitação por atributos dos frutos (aparência sem a casca, aroma, sabor e textura), além de testes de preferência e intenção de compra. Os dados foram submetidos à ANOVA $e$ Mapa de Preferência Interno. Mulheres e jovens mostraram opiniões semelhantes. As cultivares 'Preciosa' e 'Pacovan Ken' mostraram ser as mais promissoras, sendo que a primeira já pode substituir as cultivares comerciais 'Prata' $e$ 'Pacovan' sem prejudicar a sua aceitabilidade, mas a 'Pacovan Ken' ainda precisa ser melhorada quanto à aparência.

Palavras-chave: Musa spp., Mycosphaerella fijiensis Morelet; análise sensorial, testes afetivos, mapa de preferência.

\section{ABSTRACT}

The Black Sigatoka Disease is devastating to traditional banana cultivars. In Brazil, resistant cultivars are being developed but if the consumer's preference is not taken into account, the breeding program may not succeed. In this research the acceptability of four resistant cultivars was accessed in the Northeastern Brazilian region and compared to four commercial varieties. Two groups of consumers participated: young adults and housewives. Hedonic scale was applied to external appearance of bunches, global acceptance and acceptance of fruits' attributes (appearance without peel, aroma, flavor and texture). Preference and purchase intent were also evaluated. Data were submitted to ANOVA and Preference Mapping. Women and young adults showed similar opinions. 'Preciosa' and 'Pacovan Ken' cultivars showed to be the most promising ones. 'Preciosa' can already substitute the commercial 'Prata' and 'Pacovan' cultivars without impairing their global acceptability, but 'Pacovan Ken' still needs to be improved regarding its appearance.

Key words: Musa spp., Mycosphaerella fijiensis Morelet; sensory analysis, affective tests, preference mapping.

\section{INTRODUÇÃO}

O Brasil é o quarto maior produtor de banana no mundo, com mais de 7 milhões de toneladas produzida por ano (FAO, 2008), sendo o Nordeste responsável por 45\% de toda a produção do País (IBGE, 2006). Essa produção é quase toda absorvida no mercado interno, dada a importância dessa fruta na dieta da população. No entanto, as principais cultivares comercializados no país, 'Prata', 'Prata Anã', 'Pacovan', 'Maçã' e 'Nanica', são suscetíveis ao fungo Mycosphaerella fijiensis Morelet, causador do Mal da Sigatoka Negra, doença que pode causar perdas de até 100\% da produção (GASPAROTTO et al., 2003).

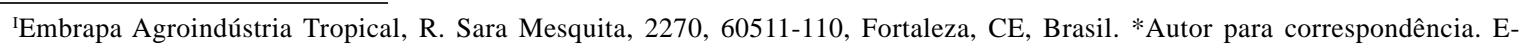
mail: deborah@cnpat.embrapa.br.

"Programa de Pós-graduação em Ciência e Tecnologia de Alimentos, Universidade Federal do Ceará (UFC), Fortaleza, CE, Brasil. IIIFaculdade de Engenharia de Alimentos, Universidade Estadual de Campinas (UNICAMP), Campinas, SP, Brasil. 
O Mal da Sigatoka Negra foi constatado no Brasil em 1998 nas áreas de produção da Amazônia, já sendo endêmico nessa região. Hoje, a doença está presente também no Mato Grosso, Mato Grosso do Sul, Paraná, litoral de Santa Catarina, Minas Gerais (CASTRO et al., 2005) e em São Paulo (FERRARI et al., 2005). Nos estados do Nordeste não se tem registro dessa doença (LOPES \&ALBUQUERQUE, 2006), mas isto pode ser questão de tempo, principalmente nos perímetros irrigados.

Como alternativa para conter o avanço da Sigatoka Negra, a Embrapa vem desenvolvendo novas cultivares resistentes à doença (SILVA et al., 2003). Entre estas, encontram-se 'Pacovan Ken', 'Preciosa', ‘Thap Maeo’ e 'Caipira'. As cultivares 'Pacovan Ken' e 'Preciosa' são ambas híbridos de 'Pacovan', uma cultivar do grupo genômico AAB, que é uma mutação da banana 'Prata' e que tem superado quase em $100 \%$ a produtividade da 'Prata'. Ambos os híbridos são tetraplóides (AAAB), oriundos do cruzamento entre a 'Pacovan' e o diplóide 'M53'(AA) e apresentam a maioria das características agronômicas semelhantes às da 'Pacovan', porém resistentes ao Mal do Panamá e às Sigatokas e com produtividade superior. Os frutos, quando maduros, apresentam casca amarela, polpa de cor creme e sabor doce, com baixa acidez (OLIVEIRA \& DANTAS, 2003; CORDEIRO et al., 2005).

O genótipo ‘Thap Maeo’ é do grupo genômico AAB e um variante da 'Mysore', uma cultivar proveniente da Tailândia. É bastante produtivo (cerca de $30 \mathrm{tha}^{-1}$ ) e apresenta frutos pequenos, semelhantes externamente à banana 'Maçã', mas de sabor diferente. É resistente às Sigatokas Amarela e Negra e ao Mal do Panamá (FANCELLI, 2003). A variedade 'Caipira', cujo nome original é 'Yangambi km 5', oriunda da África Ocidental, foi introduzida no Brasil pela Embrapa Mandioca e Fruticultura. Os frutos, curtos e grossos, possuem sabor levemente adocicado. Essa cultivar, do grupo genômico AAA, é uma variedade bastante conhecida internacionalmente pelas suas características de resistência aos principais problemas fitossanitários da cultura (FANCELLI, 2003).

No entanto, antes que os materiais resistentes sejam disponibilizados para os agricultores é necessário que a aceitabilidade de seus frutos seja avaliada pelo consumidor, pois, se esses frutos forem rejeitados, o programa de melhoramento genético não alcançará o sucesso desejado. Pelo exposto, o objetivo deste trabalho foi avaliar a aceitabilidade, na região Nordeste, de quatro cultivares de bananas resistentes à Sigatoka Negra, comparando-as com a cultivar mais comercializada nessa região ('Pacovan') e outras três cultivares comerciais de alto consumo no país, porém suscetíveis à doença.

\section{MATERIAL E MÉTODOS}

\section{Amostras}

Foram avaliadas oito cultivares de bananas (Musa ssp), sendo quatro novas cultivares resistentes à Sigatoka Negra: 'Preciosa' e ‘Pacovan Ken’ (ambas híbridas de‘Pacovan’), ‘Thap Maeo’ e ‘Caipira’; e quatro cultivares comerciais suscetíveis à doença: 'Pacovan', 'Prata', 'Prata Anã' e ‘Grand Naine' (muito semelhante à 'Nanica’, usada para exportação).

As bananas foram cultivadas no distrito de Irrigação do Baixo Jaguaribe, Ceará, em parceria com a União dos Agronegócios no Vale do Jaguaribe (UNIVALE). Os frutos foram colhidos no estádio de maturação verde, lavadose transportados até os laboratórios da Embrapa, onde foram armazenados em câmara fria, sob umidade relativa entre 80 e $85 \%$ e temperatura entre 13 a $17^{\circ} \mathrm{C}$, de acordo com a necessidade de cada variedade. Para a avaliação da aparência externa, as bananas foram amadurecidas até o grau 5 (cinco) da escala de maturação (SOTO \& BALESTERO, 1992), estádio em que os frutos apresentam cor amarela com as pontas verdes, como é costume comprar bananas no Nordeste. Para a análise dos atributos, os frutos foram amadurecidos até o grau 6 (seis), quando apresentam-se totalmente amarelos.

\section{Recrutamento e seleção dos consumidores}

Fortaleza foi a cidade escolhida para representar a região Nordeste, por ser um grande centro comercial e por ser a sede da Embrapa Agroindústria Tropical. Os consumidores foram recrutados entre alunos da Universidade Federal do Ceará, funcionários da Embrapa e moradores da vizinhança, os quais foram separados em dois grupos: i) 60 mulheres com mais de 21 anos, donas de casa que possuem o poder de decisão na hora da compra; ii) 60 adultos jovens (homens e mulheres), entre 18 e 35 anos, que frequentam academia e consomem bananas como fonte de potássio. O público foi caracterizado quanto a gênero, idade, hábitos, frequência de consumo e classe social, segundo o Critério Brasil 2008 (ABEP, 2007).

Aceitação e preferência da aparência das pencas

Uma vez que a aparência das bananas na penca é um fator de grande impacto na decisão de compra dos consumidores, dois testes de aceitação foram simultaneamente realizados: um relativo à aparência externa das pencas, e outro relativo à análise sensorial dos frutos. O teste da aparência foi montado em uma sala simulando-se uma situação de compra em banca de feira ou de supermercado. Duas pencas foram dispostas em uma bancada, em bandejas brancas e codificadas com números aleatórios de três dígitos. 
A aparência geral, cor e tamanho das bananas na penca foram avaliados utilizando-se uma escala hedônica híbrida $(15 \mathrm{~cm})$, estruturada com 9 (nove) pontos, ancorada nos extremos esquerdo e direito com os termos verbais "desgostei muitíssimo" e "gostei muitíssimo", respectivamente, similar à descrita por VILLANUEVA \& DA SILVA(2009). Para o grupo das donas de casa, foi utilizada uma escala híbrida facial desenvolvida por DA RE (2006), devido ao fato de algumas das voluntárias não serem alfabetizadas. Na ficha de avaliação, foi também incluído um teste de intenção de compra, utilizando-se uma escala de cinco pontos, como descrita por MEILGAARD et al. (1999).

Finalizado o teste hedônico, os consumidores receberam uma segunda ficha, na qual foram solicitados a ordenar as oito amostras em ordem decrescente de preferência em relação à aparência geral das pencas (teste ordenação-preferência) (MEILGAARD et al., 1999).

Aceitação global e por atributos dos frutos

Os frutos, codificados com números aleatórios de três dígitos, foram servidos ainda com casca, cortados em metades. O próprio provador retirava a casca para fazer a sua avaliação. Foi aplicado um teste de aceitação global e aceitação dos atributos aparência (banana sem casca), aroma, sabor e textura, com as mesmas escalas descritas anteriormente. $\mathrm{Na}$ ficha de avaliação, foi também incluído um teste de intenção de compra, utilizando-se uma escala de cinco pontos, como descrita por MEILGAARD et al. (1999).

As análises foram realizadas em cabines individuais climatizadas $\left(24^{\circ} \mathrm{C}\right)$, sob luz branca fluorescente. Para eliminar o sabor residual entre as amostras, foi oferecido biscoito tipo água e sal e água mineral. A ordem de apresentação das amostras foi balanceada (MACFIE et al.,1989) para minimizar os efeitos de posição das amostras.

As amostras foram analisadas em três etapas: i) análise dos frutos das quatro primeiras amostras em cabines individuais; ii) análise da aparência externa das pencas das 8 amostras em sala anexa: iii) retorno à cabine para análise dos frutos das quatro amostras restantes.

\section{Análises estatísticas}

Os dados hedônicos foram submetidos à análise de variância (ANOVA) pelo procedimento dos Modelos Lineares Generalizados (GLM) e o teste REGWQ, ao nível de 5\% de significância, para comparação das médias. Os dados de aceitação da aparência geral das pencas e aceitação global dos frutos foram também submetidos à análise multivariada de Mapa Interno de Preferência - MDPREF (MACFIE \& THOMSON, 1988). Foi utilizado o programa estatístico SAS ${ }^{\circledR}$ (SAS, 2002). Os dados de ordenaçãopreferência foram avaliados pelo Teste de Friedman, usando-se a tabela de Newell e Mac Farlane (NEWELL \& MAC FARLANE, 1987) em nível de 5\%.

\section{RESULTADOS E DISCUSSÃO}

\section{Caracterização dos consumidores}

O grupo de donas de casa era formado por mulheres entre 21 e 67 anos, sendo $35 \%$ entre 21 e 35 anos, $47 \%$ entre 36 e 50 anos e $18 \%$ acima de 50 anos. Esse grupo segmentou-se entre as seguintes classes sociais: A (10\%), B (40\%), C (35\%) e D (15\%). No grupo de adultos jovens, $65 \%$ destes possuíam entre 18 e 25 anos e $68 \%$ eram mulheres. As classes sociais predominantes (87\%) foram classes B e C. Quanto ao consumo, $49 \%$ dos entrevistados responderam que consomem banana entre uma a três vezes por semana e $35 \%$ consomem banana diariamente ou quase diariamente. Na questão "Indique o quanto você gosta ou desgosta de banana”, 66\% afirmaram gostar muito ou muitíssimo da fruta.

Aceitação da aparência externa das pencas

Pelos resultados da análise de variância realizada com os efeitos “amostra”, "grupo” e interação "grupo*amostra”, observou-se que os efeitos "grupo" e "grupo*amostra” não foram significativos $(\mathrm{P} \leq 0,05)$ para nenhum dos atributos avaliados, indicando que tanto mulheres quanto adultos jovens apresentaram a mesma aceitação quanto à aparência das pencas. Assim, os dois segmentos foram agrupados para as análises estatísticas dos testes de aceitação, preferência e intenção de compra.

Na tabela 1, observa-se que a 'Pacovan' foi a cultivar melhor aceita em relação a todos os atributos de aparência, tendo apresentado valores hedônicos próximos a "gostei muito" para aparência geral e cor e "gostei moderadamente” para o tamanho. Por sua vez, as cultivares suscetíveis do tipo 'Prata' apresentaram baixa aceitação, notadamente a 'Prata' que obteve média abaixo de 5,0 para a aparência geral, ou seja, na zona de rejeição da escala hedônica. O tamanho dos frutos da 'Prata Anã' foi tão bem aceito quanto o da 'Pacovan', porém não atingiu o mesmo nível de aceitabilidade da aparência geral, provavelmente, devido a problemas com sua cor, visto que apresentou menor média para este atributo. De fato, o consumo da banana 'Pacovan' tem crescido rapidamente no mercado de Fortaleza, em substituição à banana 'Prata' . 
Tabela 1 - Médias de aceitação ${ }^{1}$ da aparência externa das pencas e dos atributos dos frutos de quatro cultivares sensíveis e quatro cultivares resistentes à Sigatoka Negra, atribuídas por 120 consumidores da cidade de Fortaleza, CE.

\begin{tabular}{|c|c|c|c|c|c|c|c|c|}
\hline Cultivares & Aparência Global & Cor & Tamanho & Aceitação Global & Aparência & Aroma & Sabor & Textura \\
\hline 'Prata Anã' & $5,8 \mathrm{~d}$ & 5,6 bc & $6,8 \mathrm{ab}$ & eis---' & $7.20 \mathrm{a}$ & $6.56 \mathrm{a}$ & 6.43 a & $7.14 \mathrm{a}$ \\
\hline 'Prata comum' & $4,7 \mathrm{e}$ & $5,3 \mathrm{c}$ & $5,6 \mathrm{~cd}$ & $6.47 \mathrm{a}$ & $7.11 \mathrm{ab}$ & $6.67 \mathrm{a}$ & $6.41 \mathrm{a}$ & $6.79 \mathrm{ab}$ \\
\hline 'Pacovan' & 7,7 a & $7,8 \mathrm{a}$ & $7,2 \mathrm{a}$ & $6.77 \mathrm{a}$ & $7.31 \mathrm{a}$ & $6.64 \mathrm{a}$ & $6.44 \mathrm{a}$ & $7.12 \mathrm{a}$ \\
\hline 'Grand Naine' & $6,5 \mathrm{bc}$ & 5,9 bc & 6,3 bc & $6.42 \mathrm{a}$ & $6.84 \mathrm{ab}$ & $6.59 \mathrm{a}$ & $6.41 \mathrm{a}$ & $6.85 \mathrm{ab}$ \\
\hline 'Preciosa' & $6,8 \mathrm{~b}$ & $6,0 \mathrm{bc}$ & $7,1 \mathrm{a}$ & $6.51 \mathrm{a}$ & $7.10 \mathrm{ab}$ & $6.32 \mathrm{a}$ & $6.26 \mathrm{a}$ & $7.01 \mathrm{ab}$ \\
\hline 'Pacovan Ken’ & $5,8 \mathrm{~cd}$ & $5,8 \mathrm{bc}$ & $6,1 \mathrm{bc}$ & $6.92 \mathrm{a}$ & $7.07 \mathrm{ab}$ & $6.72 \mathrm{a}$ & $6.70 \mathrm{a}$ & $7.12 \mathrm{a}$ \\
\hline ‘Thap Маeо’ & $5,6 \mathrm{~d}$ & $6,2 \mathrm{~b}$ & $4,9 \mathrm{~d}$ & $6.49 \mathrm{a}$ & $7.11 \mathrm{ab}$ & $6.18 \mathrm{a}$ & $6.25 \mathrm{a}$ & $6.94 \mathrm{ab}$ \\
\hline ‘Caipira’ & 6,3 bcd & $6,0 \mathrm{bc}$ & $6,1 \mathrm{bc}$ & $5.73 \mathrm{~b}$ & $6.45 \mathrm{~b}$ & $5.57 \mathrm{~b}$ & $5.44 \mathrm{~b}$ & $6.34 \mathrm{~b}$ \\
\hline
\end{tabular}

Em uma mesma coluna, médias com letra em comum não diferem significativamente entre si a P $\leq 0,05$ pelo teste de Ryan-Einot-GabrielWelsch. ${ }^{1} 1$ = desgostei muitíssimo; 5 = nem gostei nem desgostei; $9=$ gostei muitíssimo.

Isso tem ocorrido, em grande parte, devido ao fato de a 'Pacovan' apresentar frutos grandes, firmes e vistosos, além de manter a alta resistência ao despencamento durante o transporte, característica apresentada pela 'Prata'. A 'Grand Naine', apesar de não ser muito conhecida na região Nordeste, apresentou boa aceitabilidade, atingindo média bem maior que as cultivares 'Prata'.

Das variedades resistentes, as cultivares 'Preciosa' e 'Caipira' foram as que obtiveram maiores níveis de aceitação com relação à aparência geral, tendo apresentado valores hedônicos correspondentes a "gostei ligeiramente" e "gostei moderadamente". Observa-se ainda que, assim como a banana 'Prata Anã', a aceitabilidade da aparência da cultivar 'Preciosa' pode ter sido prejudicada pela sua cor. Na verdade, quanto à cor, todas as cultivares resistentes apresentaram médias inferiores à cultivar 'Pacovan', mas não diferiram entre si. 'Pacovan Ken’ e 'Thap Maeo' foram as menos aceitas com relação à aparência da penca e a 'Thap Maeo', de frutos pequenos, não foi bem aceita quanto ao tamanho (média $<5,0$ ).

Na figura 1, os gráficos A e B representam o Mapa de Preferência Interno da aparência geral das pencas e ilustram, respectivamente, as amostras e os consumidores posicionados no espaço hedônico definido pelas duas primeiras dimensões. No gráfico $1 \mathrm{~A}$, as amostras são representadas por elipses de $95 \%$ de confiança, sendo que amostras alocadas em regiões próximas no espaço apresentam aceitação sensorial semelhante. Para a análise do Mapa de Preferência, os gráficos devem ser avaliados como figuras sobrepostas, já que os consumidores encontram-se representados por números de 1 a 120 e alocados próximos às amostras que eles preferiram e longe daquelas que menos apreciaram.

Observa-se que a Dimensão 1 apresentou uma tendência de separar as amostras de banana pelo tamanho e cor, alocando as cultivares de maior tamanho ('Pacovan', 'Pacovan Ken', 'Preciosa' e ‘Grand Naine') no lado direito do gráfico e as cultivares de menor tamanho ('Thap Maeo e Caipira') no lado esquerdo. As cultivares de menor aceitação da cor ('Prata' e 'Prata Anã’) também foram posicionadas no lado esquerdo da Dimensão 1. A maioria dos consumidores foi alocada próxima às cultivares 'Pacovan', 'Pacovan Ken', 'Preciosa' e ‘Grand Naine’, sendo que ‘Pacovan’ ficou mais próxima da maioria dos consumidores, indicando que a sua aparência obteve maior aceitação junto à maior parte dos consumidores.

Entre as cultivares resistentes à Sigatoka Negra, a 'Preciosa', de tamanho tão grande ou maior que a 'Pacovan' foi a que mais se aproximou desta última. No entanto, a cultivar resistente 'Pacovan Ken', também de frutos grandes e de mesmo nível de aceitabilidade para cor que a 'Preciosa', ficou mais distante. Assim, pode-se dizer que a aceitação da aparência global pode ter sido influenciada também pelo formato dos frutos, visto que a 'Pacovan Ken' apresenta frutos desuniformes. A banana 'Thap Maeo' também apresenta formato diferente das demais, pois é arredondada, semelhante à banana 'Maçã'. De um modo geral, os resultados mostrados no MDPREF confirmam aqueles mostrados na tabela 1. 


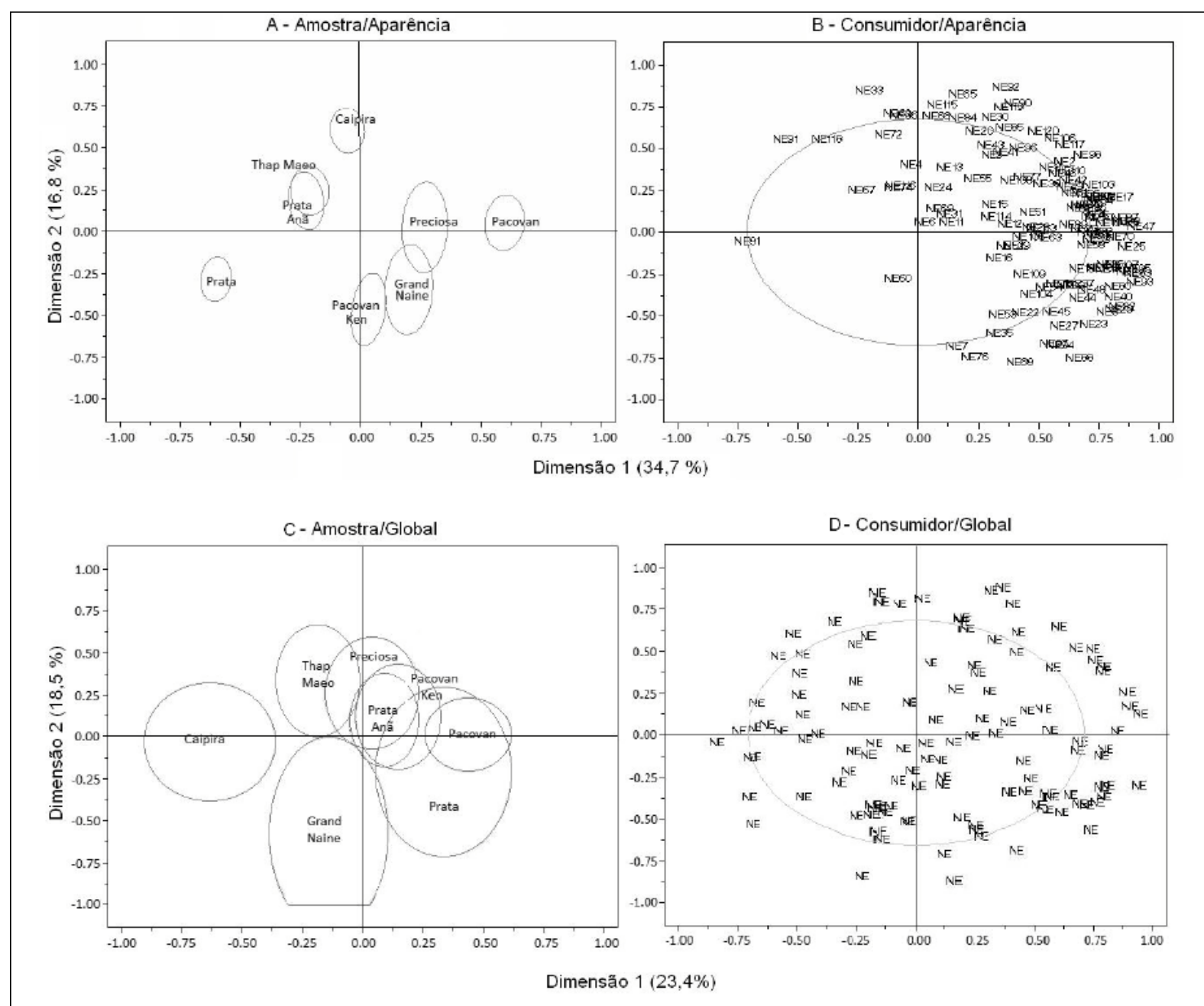

Figura 1 - Representação do MDPREF de oito cultivares de bananas, construído com as duas primeiras dimensões da Análise de Componentes Principais: amostras (A) e consumidores (B) para aceitação da aparência externa das pencas; amostras (C) e consumidores (D) para aceitação global.

No teste de Ordenação-Preferência, foram obtidos os seguintes totais de ordenação (as letras referem-se ao teste estatístico a 5\% de significância): 'Pacovan' (821a), 'Grand Naine’ (622b), 'Preciosa' (579bc), 'Caipira’(541bc), 'Prata Anã' (508bc), 'Pacovan Ken’ (491c), ‘Thap Maeo’ (468cd), 'Prata’ (363d). Observa-se que a cultivar 'Pacovan' foi de fato a mais preferida, diferindo das demais. As cultivares resistentes 'Preciosa' e 'Caipira' apresentaram preferência semelhante à da banana 'Grand Naine', a segunda mais preferida quanto à aparência. As cultivares resistentes 'Pacovan Ken' e 'Thap Maeo' foram as menos preferidas.

Quanto ao teste de intenção de compra realizado após a análise da aparência das pencas, a cultivar 'Pacovan' destacou-se por ter obtido quase $60 \%$ das respostas na categoria “certamente compraria”. A segunda colocada foi a cultivar resistente 'Preciosa', com 38\% dos consumidores afirmando que “certamente compraria” o produto e $21 \%$ “possivelmente compraria”. Porém, para a 'Pacovan Ken', somente $18 \%$ dos consumidores afirmou que “certamente compraria” as bananas. As demais cultivares resistentes, 'Caipira' e 'Thap Maeo', obtiveram respostas ainda mais desfavoráveis com relação à intenção de compra após análise da aparência das pencas.

Aceitação global e por atributos dos frutos

Pelos resultados da análise de variância realizada com os efeitos “amostra”, "grupo” e interação "grupo*amostra”, observou-se que, com exceção da aparência, o efeito "grupo" foi significativo $(\mathrm{P} \leq 0,05)$ para todas as variáveis analisadas para os frutos, sendo

Ciência Rural, v.42, n.5, mai, 2012. 
as amostras sempre melhor aceitas junto às donas de casa. Entretanto, uma vez que a interação "grupo*amostra" não foi significativa para nenhum dos atributos, pode-se assumir que o julgamento dos grupos variou apenas em intensidade do valor hedônico atribuído às amostras, não havendo discordância entre os grupos quanto à preferência das bananas degustadas: as variedades de maior preferência por um grupo foram também as mais apreciadas pelo outro. Dessa maneira, os dois segmentos foram agrupados para as análises estatísticas subsequentes.

Todas as cultivares analisadas foram igualmente aceitas quanto à aceitação global, aparência do fruto sem a casca, aroma, sabor e textura (Tabela 1), com médias situando-se entre as categorias "gostei ligeiramente” a "gostei moderadamente” da escala hedônica, com exceção da ‘Caipira’, que obteve média inferior quanto à aceitação global, aroma e sabor.

O Mapa Interno de Preferência dos dados de aceitação global (Figuras 1C e 1D) não apresentou grande segmentação das diferentes cultivares. No entanto, foi possível observar que uma maior parcela de consumidores ficou alocada no lado direito da Dimensão 1, demonstrando preferência pelas cultivares 'Pacovan', 'Pacovan Ken', 'Prata', 'Prata Anã' e 'Preciosa'. Uma minoria de consumidores demonstrou preferência pelos frutos das bananas 'Thap Maeo', 'Grand Naine' e 'Caipira', as quais ficaram situadas no lado oposto do gráfico. Esses resultados confirmam aqueles apresentados na tabela 1 e sugerem que, em termos de paladar, as variedades resistentes 'Preciosa', 'Pacovan Ken' e ‘Thap Maeo’ foram tão bem aceitas pelos consumidores do Nordeste quanto as variedades 'Pacovan' e 'Prata'.

Em relação à intenção de compra das bananas, avaliada após a degustação dos frutos, cerca de $40 \%$ dos consumidores afirmaram que certamente comprariam as bananas das variedades 'Pacovan', 'Prata Anã', 'Pacovan Ken’ e 'Grand Naine’. De fato, em termos de paladar, a 'Pacovan Ken' superou a 'Pacovan’ na intenção de compra. Embora inferior à 'Pacovan Ken', a cultivar ‘Thap Maeo' também obteve bons resultados, com $28 \%$ das respostas em “certamente compraria” e 24\% em "possivelmente compraria”. A 'Caipira', por outro lado, apresentou maior índice de rejeição, com 35\% dos respondentes afirmando que não comprariam o fruto, com um grau de certeza entre "possivelmente" e "certamente".

\section{CONCLUSÃO}

Os resultados revelam uma grande satisfação dos consumidores de Fortaleza, representantes do Nordeste, com as características de aparência da penca, tamanho dos frutos, cor da casca, aparência do fruto descascado, aroma, sabor e textura da cultivar 'Pacovan'. A maior diferença de aceitabilidade sensorial entre a 'Pacovan' e as bananas resistentes à Sigatoka Negra, como a 'Preciosa', 'Pacovan Ken’ e 'Thap Maeo', ocorreu na aparência da penca, e não em atributos associados à palatabilidade dos frutos.

Entre as variedades resistentes, a 'Preciosa' obteve os melhores resultados junto aos consumidores quanto à aparência da penca. Por outro lado, considerando-se apenas os atributos sensoriais dos frutos, a cultivar 'Pacovan Ken' foi tão bem aceita quanto a 'Pacovan'. Estes resultados tornam essas duas variedades as mais promissoras para futuros programas de melhoramento de bananas resistentes à Sigatoka Negra para o Nordeste brasileiro. A cultivar 'Preciosa' pode substituir as variedades comerciais 'Prata' e ‘Pacovan' sem prejudicar sua aceitabilidade, porém a ‘Pacovan Ken’ precisa ainda ser melhorada quanto à sua aparência.

\section{AGRADECIMENTOS}

Os autores agradecem à União dos Agronegócios no Vale do Jaguaribe (UNIVALE) e à Fazenda FRUTACOR, pela instalação do experimento, à Coordenação de Aperfeiçoamento de Pessoal de Nivel Superior (CAPES), pela concessão da bolsa de estudos ao Banco do Nordeste (BNB) e ao Fundo de Desenvolvimento Científico e Tecnológico (FUNDECI), pelo financiamento da pesquisa.

\section{COMITÊ DE ÉTICA}

Parecer CEP Unicamp - 133/2007.

\section{REFERÊNCIAS}

ABEP - Associação Brasileira das Empresas de Pesquisa. Critério padrão de classificação econômica Brasil/2008, 2007. Disponível em: <http://www.abep.org/novo/Content.aspx? ContentID=302>. Online. Acesso em: 25 fev. 2008.

CASTRO, M.E.A. et al. First report of Black-Sigatoka in the State of Minas Gerais, Brazil. Fitopatologia Brasileira, v.30, n.6, p.688, 2005. Disponível em: <http://dx.doi.org/10.1590/ S0100-41582005000600018>. Acesso em: 19 nov. 2011. doi:10.1590/S0100-41582005000600018.

CORDEIRO, Z.J.M. et al. 'Preciosa': variedade de banana resistente àSigatoka-Negra, Sigatoka-Amarela e ao Mal-doPanamá.Fitopatologia Brasileira, v.30, n.3, p.316, 2005. Disponível em: <http://dx.doi.org/10.1590/S010041582005000300022>. Acesso em: 19 nov. 2011. doi:10.1590/ S0100-41582005000300022.

DA RE Desempenho de crianças em testes sensoriais discriminativos e afetivos com escalas híbridas ilustradas. 
2006.150f. Tese (Doutorado em Alimentos e Nutrição) Faculdade de Engenharia de Alimentos, Campinas, SP.

FANCELLI, M. Cultivo da banana para o estado do Amazonas. Cruz das Almas: Embrapa Mandioca e Fruticultura, 2003.4p. (Sistema de produção, 6).

FAO. Agricultural Data, 2008, FAOSTAT, Agricultural Production. Disponível em: <http://faostat.fao.org/site/339/ default.aspx>.Online. Acesso em: 25 jan. 2011.

FERRARI, J.T. et al. Ocorrência de Sigatoka-Negra em São Paulo. Arquivos do Instituto Biológico, v.72, n.1, p.133134, 2005. Disponível em: <http://www.biologico.sp.gov.br/ rev_arq.php?vol=72\&num=1>. Acesso em: 15 jan. 2011.

GASPAROTTO, L. et al. Sigatoka-negra: Situação atual e avanços obtidos. In: SIMPÓSIO BRASILEIRO SOBRE BANANICUlTURA, 5., 2003, Paracatu. Anais... Cruz das Almas: Nova Civilização, 2003. p.28-34.

IBGE. 2006. Sistema IBGE de recuperação automática SIDRA. Disponível em: <http://www.sidra.ibge.gov.br/bda/ tabela/protabl.asp? $\mathrm{c}=1730 \& \mathrm{z}=\mathrm{t} \& \mathrm{o}=1 \& \mathrm{i}=\mathrm{P}>$. Online. Acesso em: 25 jan. 2011.

LOPES, E.B.; ALBUQUERQUE, I.C. Levantamento fitopatológico de doenças da bananeira com ênfase à Sigatoka Negra (Mycosphaerella fijensis Morelet) nos municípios produtores de banana da Paraíba. 2006. Disponível em: <http://www.emepa.org/inform/ sigatoka_0.htm>. Acesso em: 26 jan. 2009.

MACFIE, H.J. et al. Designs to balance the effect of order of presentation and first-order carry-over effects in hall tests. Journal of Sensory Studies, v.4, p.129-148, 1989. Disponível em: <http://onlinelibrary.wiley.com/doi/10.1111/ j.1745-459X.1989.tb00463.x/abstract>. Acesso em: 19 nov. 2011. doi:10.1111/j.1745-459X.1989.tb00463.x.
MACFIE, H.J.; THOMSON, D.M.H. Preference mapping and multidimensional scaling. In: PIGGOT, R.J. (Ed.). Sensory analysis of food. New York: Elsevier, 1988. p.341-409.

MEILGAARD, M.R. et al. Sensory evaluation techniques.Boca Raton: CRC, 1999. 382p.

NEWELL, G.J.; MAC FARLANE, J.D. Expanded tables for multiple comparison procedures in the analysis of ranked data. Journal of Food Science, v.52, n.6, p.1721-1725, 1987. Disponível em: <http://onlinelibrary.wiley.com/doi/10.1111/ j.1365-621.1987.tb05913.x/abstract>. Acesso em: 19 nov. 2011. doi:10.1111/j.1365-2621.1987.tb05913.x.

OLIVEIRA, A.A.R.; DANTAS, J.L.L. Variedades melhoradas de fruteiras, 2003. Disponível em: <http:// www.paginarural.com.br/artigo/691/variedades-melhoradas-defruteiras>. Acesso em:16 jul. 2010.

SAS (Statistical Analysis System) for Windows, Versão 9.1. Carry, USA: Microsoft Corporation, 2002.CD-ROM.

SILVA, S.O. et al. Programa de Melhoramento de Bananeira no Brasil - Resultados Recentes. Cruz das Almas: Embrapa Mandioca e Fruticultura, 2003.36p. (Documentos. ISSN 1516-5728. n.123).

SOTO BALESTERO, M. Bananos: cultivo y comercialización. San Jose, Costa Rica: Litografia e Imprensa LIL, 1992. 674p.

VILLANUEVA, N.; DASILVA, M. Comparative performance of the nine-point hedonic, hybrid and self-adjusting scales in the generation of internal preference maps. Food Quality and Preference, v.20, p.1-12, 2009. Disponível em: <http:// w w w. sciencedirect.com/science/article/pi i / S0950329308000803>. Acesso em: 19 nov. 2011. doi:10.1016/ j.foodqual.2008.06.003. 